\title{
Evaluation of the Foamability Potential of a Novel Biosurfactant Using the Solution to Advective-Diffusive Transport Model in Porous Media With a Linear Adsorption Trend
}

\author{
Mumuni Amadu ${ }^{1}$, Adango Miadonye ${ }^{2}$ \\ ${ }^{1}$ Department of Process Engineering and Applied Science, Dalholusie University, Halifax, Canada \\ ${ }^{2}$ Faculty of Egineering and Technology, Cape Breton University, Canada \\ Correspondence: Mumuni Amadu, Department of Process Engineering and Applied Science, Dalholusie University, \\ Halifax, Canada. E-mail:mumuniamadu@hotmail.com or mm846771@dal.ca
}

$\begin{array}{lc}\text { Received: April 5, } 2018 & \text { Accepted: April 26, } 2018 \quad \text { Online Published: April 27, } 2018 \\ \text { doi:10.5539/ijc.v10n2p56 } & \text { URL: https://doi.org/10.5539/ijc.v10n2p56 }\end{array}$

\begin{abstract}
While geological sequestration of anthropogenic carbon dioxide is a technically and economically viable option for reducing emissions to the level required to avoid the predicted 2 degrees Celsius increase of atmospheric temperature by the end of this century, efficient sequestration planning is vital for the achievement of this goal.

The petroleum industry has used conventional surfactants in enhance oil recovery projects aimed at prolonging the life span of a field, thereby increasing ultimate reserves. Notable among these is the use of surfactants for injected gas relative mobility control. Therefore, the potential for carbon dioxide mobility control in saline aquifers using surfactant alternating gas injection is huge, given the rich experience that can be tapped from the petroleum industry practice.

Considering the expected surfactant loses in surfactant-enhanced geological sequestration similar to that encountered in the petroleum industry, this paper has used the analytical solution to advective diffusive equation that exists in the literature with a linear adsorption model where, adsorption has been used to predict trends in minimum pressure drop required for foam generation. The greatest utility of this work lies in the fact that the analytical solution is related a linear adsorption model related to a novel surfactant found from biological and hydrocarbon sources of geologic origin. This paper, therefore, extends the work of linear adsorption models for this novel surfactant aimed at exploring improved oil recovery potentials; in addition to exploring its potential for efficient geological carbon storage in saline aquifers.
\end{abstract}

Keywords: surfactant, dispersivity, advective, linear model, adsorption

\section{Introduction}

The exponential trend in energy consumption both at domestic and industrial levels since the industrial revolution has resulted in unprecedented release of anthropogenic carbon dioxide into the atmosphere. Evidence abounds that the pre-industrial level concentration of carbon dioxide $280 \mathrm{ppm}$ (Monroe, 2013) has increased to current a current concentration of 370 moles per liter with a projected increase to a level of 600 moles/L this century (Ziska \& Caulfield, 2000). Carbon concentration in the atmosphere is believed to be increasing at a rate of 2-3 ppm/yr meaning the critical value will be reached about 30 years from year 2007. (Global Warming, 2009) The effect of this dramatic increase in carbon dioxide concentration, which is a greenhouse gas is seen in the form of eminent global warming (Solomon et al., 209).

Considering the exponential trend in carbon dioxide emission, the prime goal of geological sequestration is to enhance volumetric sweep efficiency (Verma, 2015), which is defined as the fraction of subsurface pore volume that is occupied by injected carbon dioxide at abandonment. To achieve this goal, higher sweep efficiencies during gas injection must be emphasized. The industry with the greatest experience in subsurface fluid injection is the petroleum industry, which has implemented surfactant enhanced oil recovery schemes since the beginning of the petroleum industry. Quite recently, Vitoonkijvanich et al., (2015), studied surfactant enhanced geological carbon storage using stream tubes. Hirasaki et al. (1977) (Hirasaki, Szafranski, Miller, \& Wade, 1977) also studied surfactant enhanced remediation of aquifers with dense non-aqueous phase fluids. In all cases, the researchers arrived at the conclusion that surfactant enhanced injection can significantly increase storage efficiency. 
In the surfactant enhanced geological storage, surfactants are used to generate foam to improve injected gas relative mobility leading to improved gas injection sweep efficiency. In the water-gas alternating scheme (Zekri et al., 2011), a surfactant solution can be injected to sweep the original brine in the aquifer. This injection will can be achieved at higher sweep efficiency due to miscible injection (Aghdam et al., 2013). During the gas injection stage, the presence of surfactant solution in the aquifer will result in foam generation, which increase injected gas dynamic viscosity to improve sweep efficiency. However, in the application of foam enhanced oil recovery, the experienced gathered by researchers is that below the critical surfactant concentration no foam will be generated (Vitoonkijvanich et al., 2015). Also, a minimum pressure drop is required to cause foam generation and mobilization in porous media (Frank, et al., 1985). This minimum pressure drop is linked to the interfacial tension between injected gas and surfactant solution.

However, while surfactants have proven to be vital to enhanced oil recovery, one fundamental problem related to their field applications is their adsorption on rock minerals, which constitutes a huge loss to the petroleum industry (Gogoi, 2011). Consequently, immense research efforts to understand the kinetics of adsorption (Gandomkar \& Kharrat, 2013) coupled with advanced mathematical modeling of fluid flow in porous media has resulted in the availability of several adsorption models (Kwok et al., 1995) in addition to models of dispersive and advective flow in porous media. The latter models integrate adsorption models to describe the evolution of surfactant concentration in porous media with a fundamental parameter called the retardation (Cameron, 1977).

The petroleum industry is used to using conventional surfactant for tertiary oil recovery projects (Schramm L., 2009). However, recent studies have evaluated the potential of a biosurfactant, Ziziphus Christis, which is also found in oil reservoir for enhance oil recovery projects (Safian-Boldaji et al., 2013). The motivation for these studies stems from the fact that these surfactants are naturally available and can be extracted for commercial purposes. Considering the availability of the above mentioned valuable mathematical resources, it is my assertion that since the minimum pressure drop is linked to interfacial tension, which in turn is linked to surfactant concentration, the analytical solution to a mathematical model that integrates the appropriate adsorption model can help predict the evolution of the minimum pressure drop required for foam generation in a porous medium. Consequently, the analytical solution to a mathematical using adsorption kinetic parameters that describe the linear adsorption model where Ziziphus Christis is used for experiments will be an extension of the investigation of this surfactant not only for tertiary oil recovery projects but also for enhanced geological storage of anthropogenic carbon dioxide. This objective will be pursued in this paper.

\section{Literature Review}

\subsection{Previous Researches in Geological Carbon Storage}

Since the beginning of geological storage research activities several researches have been done to understand how efficient a commercial or large-scale project can be embarked upon. Famous among these researches are those related to relative permeability (Chen at al., 2014;Muller, 2011),convective transport of injected carbon dioxide (Sun et al., 2015), wettability changes associated with carbon dioxide injection into saline aquifers (Kim et al. 2012;Farokpoor et al., 2013) , convective instability (Lindeberg \& Wessel-Berg, 1997) and supercritical carbon dioxide interfacial tension variation with salinity, temperature and pressure (Bachu \& Bennion, nterfacial Tension between $\mathrm{CO}_{2}$, Freshwater, and Brine in the Range of Pressure from (2 to 27) MPa, Temperature from (20 to 125) ${ }^{\circ} \mathrm{C}$, and Water Salinity from (0 to $\left.334000) \mathrm{mg} \cdot \mathrm{L}^{-1}, 2009\right)$. In addition, researches aimed at understanding carbon dioxide solubility in saline aquifers under supercritical conditions (Pruess \& García, 2002), which constitutes solubility trapping (Nghiem, et al., 2009), has also been carried out. It is the view of the present paper that solubility trapping in saline aquifers, which is very essential for bulk carbon immobilization will be most successful following efficient displacement of formation brine and optimization of storage efficiency. To achieve this goal requires learning from the experience of the petroleum industry that has used foam enhanced gas flooding to improve oil recovery during tertiary oil recovery phases.

\subsection{Physiochemical Processes Associated With Porous Media Fluid Transport With Adsorption}

The integrated physiochemical processes of interest in this paper are advective dispersive and adsorption. Advective transport is the flow of a fluid in bulk while dispersion is the spreading of a substance in stream in a direction. The major causes of hydrodynamic dispersion are variation of flow velocity with pores and heterogeneities in the porous medium domain such as higher permeability zones. In this regard, the mechanical dispersion coefficient is defined as the product of Dispersivity, which is a length scale dependent (Peeters \& Hofmann, 2015) and velocity. Generally, Dispersivity measures the spreading of a contaminant over a given length of flow and it is governed principally by the hydraulic properties of the porous system in addition to being the sum of molecular and mechanical dispersion. In flow systems with contaminant transport, the presence of dispersion causes the solute front to move faster than the case where advective transport alone was responsible for contaminant transport (Cheremisinoff, 2017). Molecular diffusion occurs due to variation in concentration gradient of species in the direction of flow.

Adsorption is a physiochemical process that is caused by van der Waal interaction (Chen \& Al-Saidi, 2012) between a 
solid surface and ions or molecules or by electrostatic attraction between an ion or a molecule and a solid surface. The electrostatic origin of adsorption is directly linked to the development of the electric double layer through ionization of surface functional groups at prevailing aqueous solution $\mathrm{pH}$ (Frank, et al., 1985). Generally, adsorption of contaminants onto aquifer materials causes an imminent retardation or slowing relative to average interstitial or groundwater flow (Wexler, 1992), which has the potential to reduce water solubility of contaminants; in addition to controlling contaminant volatility (Patil \& Chore, 2014) and biodegradability.

\subsection{Global Availability of Biosurfactant (Ziziphus Christis) and Potential for Enhanced Oil Recovery Projects}

In the application of surfactants to enhanced oil recovery in the petroleum industry, economic and environmental constraints must be met due to adsorption, proper interfacial tension reduction and oil recovery efficiency (Spildo et al., 2012). The environmental aspect of the constraint, which emphasises on biodegradability of spent surfactant, has aroused interest in the use of biosurfactant. Consequently, Pordel et al., (2012) investigated the use of extracted surfactant from zyziphus spina christi in oil recovery by means of Amott wettability index. In addition, core displacement tests have been performed to determine sweep efficiency of the extracted surfactant from leaves of zyziphus spina christi at different concentration (Zendehboudi et al., 2103). Finally, they, Zendehboudi et al., (2013), developed thermodynamic modeling for the adsorption of mentioned surfactant onto carbonate rock surface. In addition to these cited works involving the use of biosurfactant, Rahmati et al., (2015), have also investigated experimentally the effect of Natural Leaf-derived Surfactants on Wettability Alteration and interfacial tension reduction in water-oil system for enhanced oil recovery application

Ziziphus species (Rhamnaceae) occur in warm-temperate and sub-tropical regions around the world, and they are commonly used as herbs in folk medicine for the treatment of various diseases, such as digestive disorders, weakness, liver complaints, obesity, urinary problems, diabetes, skin infections, fever, diarrhea, and insomnia (Abdel-Zaher et al., 2005). Ziziphus Spina-Christi, for instance, is a wild evergreen tree characterized by spiny branches and small orange fruits that is native to sub-tropical eastern Africa, the Middle East, and the Indian subcontinent, but also widespread in northwestern Africa. In the Middle Eastern regions, leaves of Z. Spina-Christi are harvested from the wild or from cultures and sold as herbal medicine. In Iran, the province of Khuzestan is known for the extensive cultivation of Z. spina-christi, and leaves are used in Iran as a natural detergent and shampoo due to the saponin content (Bozicevic et al., 2017).

Ziziphus Christis also occurs in connection with oil deposits in Iran (Ahmadi \& Shadizadeh, 2012)

\subsection{Relevant Literature Based Models for the Present Work}

In the petroleum industry, foam has been used for two primary purposes. They are, its used in connection with gas diversion or mobility control during gas injection for enhanced oil recovery (Gauglitz et al., 2002) and its used for acid diversion in connection with matrix acidization in hydrocarbon well stimulation (Schramm, 1994; Terdre, 2003). In the former case, the generation of foam in oil reservoirs serve to alleviate the huge dynamic viscosity contrast between injected gas and resident oil, which is the principal cause of viscous fingering and viscous instability (Didier, 1992; Moortgat, 2016).

However, while surfactants have proved to be vital inputs in sustaining oil production beyond secondary water flooding schemes, one fundamental problem connected to their field applications is their adsorption on rick minerals, which constitutes a huge loss annual basis (Fabíola, et al., 2007). Consequently, numerous researches designed to understand adsorption trends has led to models of adsorption isotherms, which seek to quantify surfactant adsorption as a function of concentration at a given temperature. In the literature, several adsorption isotherms have emerged, famous among which are the Freundlich model (Freundlich, 1906), Langmuir (Barati et al., 2016), Temkin model (Temkin \& Pyzhev, 1940) and the linear model (Ruthven, 1982). Apart from these, there is the general model that gives isotherms of different shapes depending on the values of the parameters of the model ( $\mathrm{Li}$ et al., 2016). The linear model, which is similar to Henrys law is given as (Ruthven, 1982):

$$
\Gamma=K_{H} C
$$

Where:

$\Gamma=$ Amount absorbed in $\mathrm{mg} / \mathrm{gm}$ of rock

$K_{H}=$ A constant of the linear isotherm- $\mathrm{L} / \mathrm{m}^{2}$

$C=$ Concentration of surfactant in the aqueous phase-?

While research has been going on to understand surfactant adsorption, the conditions under which foam generation and mobilization will occur in porous media has also received a great deal of attention. Consequently, there is a consensus 
among researchers that there is minimum pressure drop required for foam generation (Gauglitz et al., 2002; Chena et al., 2005; Barati et al., 2016). This minimum pressure drop is linked to the petrophysical properties of the porous medium as (Gauglitz et at., 2002):

$$
\Delta P^{\min }=\frac{2 \sigma}{L} \sqrt{\frac{\phi}{K}}
$$

Where:

$\Delta P^{\mathrm{min}}=$ Minimum pressure drop required for foam generation-Pa

$\sigma=$ Interfacial tension between gas and brine $\mathrm{N} / \mathrm{m}$

$K=$ Absolute permeability of porous sample- $\mathrm{m}^{2}$

$\phi=$ Porosity-fraction

In the petroleum and chemical engineering industries, the concentration dependence of surface tension regarding surfactants concentration has been studied (Eastoe \& Dalton, 2000). The dependence of surfactant solution surface tension on adsorption is given as (Vizquez et al.,1997):

$$
\sigma-\sigma_{0}=R T \Gamma^{\infty} \ln \left(1-\frac{\Gamma}{\Gamma^{\infty}}\right)
$$

Where:

$\sigma=$ Surface tension of surfactant solution- $\mathrm{mN} / \mathrm{m}$

$\sigma_{o}=$ Initial value of surface tension- $\mathrm{mN} / \mathrm{m}$

$R=$ Universal value of surface tension

$T=$ Absolute temperature

$\Gamma=$ Adsorption-mols $/ \mathrm{m}^{2}$

$\Gamma^{\infty}=$ Equilibrium adsorption-mols $/ \mathrm{m}^{2}$

Under transient conditions, the surface tension is given as:

$$
\frac{\sigma-\sigma_{e}}{\sigma_{o}-\sigma_{e}}=\exp \left(-b t^{\frac{1}{2}}\right)
$$

Where:

$\sigma_{e}=$ Equilibrium value of surface tension- $\mathrm{mN} / \mathrm{m}$

$b=$ Concentration dependent constant

$t=$ Time-min

For a two-component system, the relationship between surface tension and excess adsorption at a given temperature and pressure is given as (Gildnyi et al., 1976):

$$
\Gamma_{e x c}=\frac{1}{R T}\left(\frac{\partial \sigma}{\partial \ln (a)}\right)_{T P}
$$

Where:

$\Gamma_{e x c}=$ Excess adsorption and at the interface over what will prevail assuming the bulk concentration continued to the interface-moles $\mathrm{m}^{2}$

$R=$ Universal gas constant

$T=$ Absolute temperature-K

$a=$ Activity of surfactant 
Equations (1), (3), (4) and (5) provide the opportunity to see a link among adsorption, surface tension and concentration while Eq. (2) links the minimum pressure drop to adsorption through the effect of surfactant adsorption on surface tension. Also, the minimum pressure drop equation is linked to interfacial tension. Therefore, any solution to a mathematical model that integrates adsorption model will provide the opportunity to link surfactant concentration to both the minimum pressure drop required for foam generation and the interfacial tension between carbon dioxide and surfactant solution. What is more, considering dynamic viscosity and surface tension dependence on solution components, Li et al., (2016), used adsorption experiments to determine surface tension and dynamic viscosity dependence of surfactant solution. Thus, considering flow model being a function of space and time, the concentration of surfactant solution versus time and space can be linked to interfacial tension and to the minimum pressure drop for foam generation in a porous medium under surfactant enhanced geological carbon storage. The following section will be devoted to the required mathematical modeling for achieving this objective.

\section{Mathematical Model}

\subsection{Shell Mass Balance Approach}

The saline aquifer under carbon dioxide injection is schematized in Fig. 1.The aquifer is assumed to have an infinite lateral extent with an infinite radius. It has a geological storage capacity measured by porosity $\phi$ and an absolute permeability of $\mathrm{K} \mathrm{m}^{2}$. The depth of storage is chosen to be greater than $800 \mathrm{~m}$ meters to ensure supercritical conditions of storage (Shariatipour et al., 2012). In this geological storage projects, alternating surfactant solution and carbon dioxide injection would be assumed to be the most optimum strategy for enhancing gas injection sweep efficiency considering the relative mobility control aspect. For mass balance, a differential element of lateral extent, delta $r$, is considered. This element has a cross section area of $A_{c}$. normal to the flux of injection. In the petroleum engineering literature, linear horizontal flow (Al-Rbeawi \& Tiab, 2014) for extensive reservoir have been used for analysis and this paper will endeavor to adopt this type of flow.

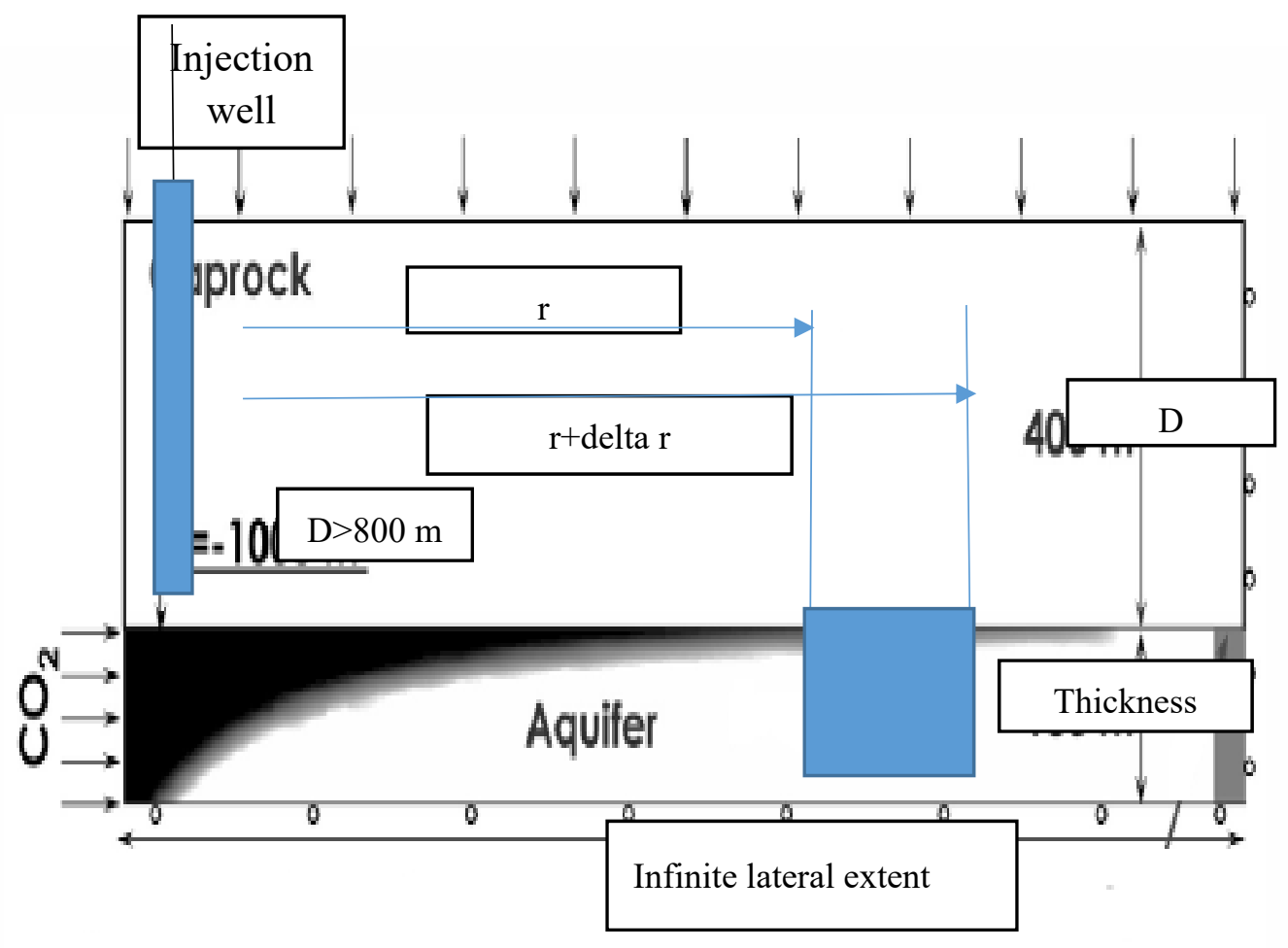

Figure 1. Schematics of Saline aquifer with differential element

The injector is first used to inject surfactant solution to displace formation brine. During the second phase, which is the gas injection phase, carbon dioxide will be injected to facilitate the generation of foam, which will improve the relative mobility of the injected gas. The volume of the differential element is calculated as:

$$
V=2 \pi r H \Delta r
$$

$A_{c}=$ Area opened to injection flux 
$V=$ Volume of differential element

$\Delta r=$ Lateral extent of differential element

$H=$ Thickness of injection interval

3.2 Convective Diffusive Transport Modeling

In this mathematical formulation, a differential volume element of a saline aquifer at a depth typical of carbon sequestration is considered in relationship to the fundamental principle of mass balance. The following are the assumptions:

1. The aquifer is extensive and has a competent cap rock above the injection interval that has enough threshold capillary pressure to sustain the upward migration of injected gas

2. The principal hydraulic processes that control the transport of surfactant in the saline aquifer are advection and dispersion with surfactant adsorption being modeled by the linear adsorption theory

3. Injection of surfactant solution will precede that of gas to facilitate foam generation for gas relative mobility control

Base on the assumptions, the convective and diffusive terms, which constitute the mass flux of injected surfactant solution, are given as (See Figure 1):

$$
q C-D \phi \frac{\partial C}{\partial r}
$$

$D_{e f f}=D \frac{\phi}{\tau}, q, C, \phi, D, D_{e f f}, r, \tau$

are flow rate, concentration of aqueous solution, porosity of aquifer formation, diffusion coefficient, effective diffusion coefficient radial distance and tortuosity respectively

$D=$ Diffusion in the absence of tortuosity factor $-\mathrm{m}^{2} \mathrm{~s}^{-1}$

Consequently, the rate of mass entry into the differential volume element at the location, $r$ is:

$$
\left(q C-2 \pi r H D_{e f f} \phi \frac{\partial C}{\partial r}\right)_{r}
$$

Where:

The rate at which mass leaves the differential volume element at the exit is:

$$
\left(q C-2 \pi r D \phi \frac{\partial C}{\partial r}\right)_{r+\Delta r}
$$

$U \frac{\partial C}{\partial r}$ and $-D_{e f f} \frac{\partial C}{\partial r}$ are radial advective and diffusive fluxes respectively

$U$ is the superficial velocity of flow

The rate of mass accumulation in the differential element will be given by:

$$
2 \pi r H \Delta r \phi \frac{\Delta C}{\Delta t}
$$

Where:

$H=$ Thickness of aquifer or injection interval

$H=$ Thickness of aquifer residual formation water saturation behind front-fraction

The application of the statement of mass balance, for diffusive-advective transport leads to the following: 


$$
\frac{2 \pi H\left(r D \frac{\partial C}{\partial r}\right)_{r}-\pi H\left(r D \phi \frac{\partial C}{\partial r}\right)_{r+\Delta r}}{2 \pi r \Delta r H}+\frac{\left.q C\right|_{r}-\left.q C\right|_{r+\Delta r}}{2 \pi r H}=\phi \frac{\Delta C}{\Delta t}
$$

Hence:

$$
\phi \frac{\partial C}{\partial t}=\frac{1}{r} \frac{\partial}{\partial r}\left(r \frac{\partial C}{\partial r}\right)-\frac{U}{r} \frac{\partial C}{\partial r}
$$

Where:

\subsection{Adsorption Modeling}

$$
U=\frac{q}{2 \pi r H}
$$

The volume of solid associated with solid in the differential element is given as

$$
2 \pi r H \Delta r(1-\phi)
$$

The mass of this element is:

$$
2 \pi r H \Delta r(1-\phi) \rho_{r}
$$

Where:

$\rho_{r}=$ Is the mean density of rock

The adsorption is measured as milligram of surfactant per gram of solid phase. Dividing the adsorption by density, therefore, gives surfactant adsorption per unit volume of solid phase. Thus:

$$
\frac{\frac{S_{m}}{g_{r}}}{\rho_{r}}=\Gamma
$$

$S_{m}=$ Mass of surfactant adsorbed on rock in the differential volume-milligram

$g_{r}=$ Weight of rock in the differential element-gram

$\Gamma=$ Weight of surfactant adsorbed pet unit volume of rock-milligram per volume of rock

The rate of adsorption of surfactant becomes:

$$
R S A=\frac{\partial \Gamma}{\partial t}
$$

Where:

$R S A=$ Rate of surfactant adsorption-milligram per volume of rock per unit time

The complete mass balance consisting of transport and adsorption becomes: Solution Assumption.

$$
\phi \frac{\partial C}{\partial t}+\frac{\partial \Gamma}{\partial t}=\frac{1}{r} \frac{\partial}{\partial r}\left(r \frac{\partial C}{\partial r}\right)-\frac{U}{r} \frac{\partial C}{\partial r}
$$

\subsection{Linear Flow Model}

1. The aquifer is hydraulically extensive, meaning that the lateral extent is very big compared with the vertical thickness and this causes pressure gradients in the vertical direction to be negligible 
2. Vertical flow is negligible

3. Dispersivity in the vertical direction is negligible

4. Geological sequestration of anthropogenic carbon dioxide will be confined to ultra-saline aquifers with hyper salinities

Fig. 2 shows a sketch of the stream lines for flow in the vicinity of a well bore. Based on this model of stream lines, which appear elliptical, a differential element for control volume analysis shows that a constant cross-sectional area of flux can be approximated. This causes the radial advective-dispersive equation to be reasonably approximated by a one dimensional of linear flow as (Chrysikopoulous et al., 1990).

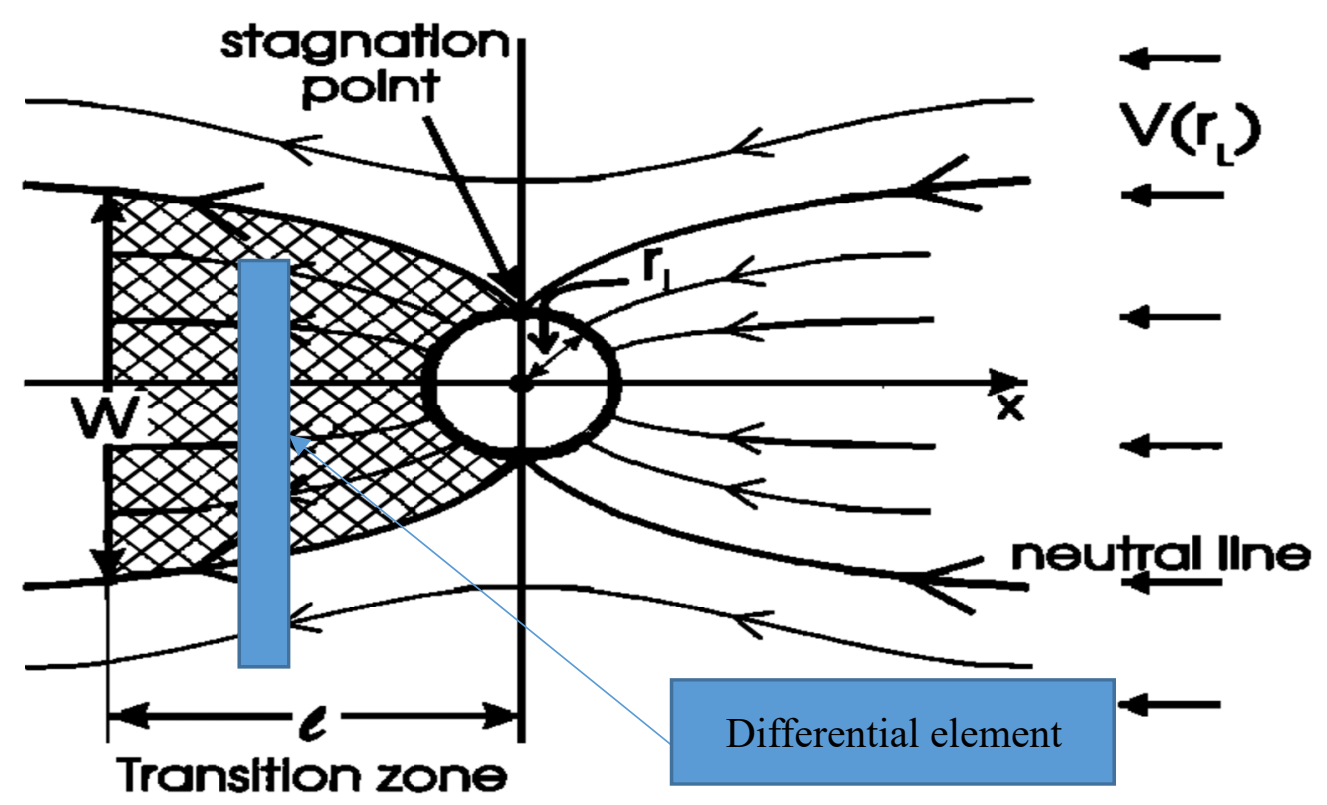

Figure 2. Schematic of linear model (Zlotnik \& Logan, 1996)

In light of the flow schematics of Figure 2, a one-dimensional advective-diffusive transport with adsorption component has been mathematically described elsewhere (Lapidus \& Amundson, 1952): The equivalent form of their equation can be written a:

$$
\phi \frac{\partial C}{\partial t}+\frac{\partial \Gamma}{\partial t}=D \frac{\partial^{2} C}{\partial x^{2}}-U \frac{\partial C}{\partial x}
$$

A linear isotherm under isotropic exchange conditions may be generally classified as either a reversible or irreversible reaction. The simplest form is the linear isotherm which represents an irreversible system trend described by the following model: (Ogata, 1964)

$$
\frac{\partial \Gamma}{\partial t}=K C
$$

Where:

$C=$ Liquid phase concentration equal to the concentration of surfactant-mass per unit volume

$\Gamma=$ Solid phase adsorption-mass per unit volume of solid

$K=$ A constant of the linear isotherm- s $^{-1}$

$$
\phi \frac{\partial C}{\partial t}+K C=D \frac{\partial^{2} C}{\partial x^{2}}-U \frac{\partial C}{\partial x}
$$

Hence: 


$$
\frac{\partial C}{\partial t}+\frac{K}{\phi} C=\frac{D}{\phi} \frac{\partial^{2} C}{\partial x^{2}}-\frac{U}{\phi} \frac{\partial C}{\partial x}
$$

This is a second order liner partial differential equation.

\section{Analytical Solution}

4.1 Solution with Diffusion and Advective Transport

Equation (21) is a second order linear partial differential equation in light of the invoked linear adsorption model. The following are the boundary conditions for solution of the concentration evolution of surfactant in the saline aquifer:

$$
\begin{aligned}
& C(0, t)=C_{0} \\
& C(r, 0)=0 \\
& C(\infty, t)=0
\end{aligned}
$$

The Laplace transform of Eq. (21) gives:

$$
\begin{gathered}
\frac{\phi}{D} \frac{\partial C}{\partial t}+\frac{K}{D} C=\frac{\partial^{2} C}{\partial x^{2}}-\frac{U}{D} \frac{\partial C}{\partial x} \\
\frac{d^{2} C(s)}{d x^{2}}-\frac{U}{\phi} \frac{d C(s)}{d x}-\xi_{s} C(s)-\psi C(s)=0 \\
\frac{d^{2} C(s)}{d x^{2}}-\lambda \frac{d C(s)}{d x}-(\xi s+\psi) C(s)=0
\end{gathered}
$$

Where:

$S=$ Laplace transform variable

$$
\begin{aligned}
& \lambda=\frac{U}{D} \\
& \xi=\frac{\phi}{D} \\
& \psi=\frac{K}{D}
\end{aligned}
$$

The solution to this equation was given by Ogata and Banks (1961) as:

$$
C(x, t)=J \frac{2}{\sqrt{\pi}} \exp (-\mu t) * \exp (\mu t) \frac{\sqrt{\pi}}{2}\left(\frac{1}{2}\right)\left[\begin{array}{l}
\exp \left\{\frac{U}{2 D} x\left[1-(1+\omega)^{1 / 2}\right]\right\} \operatorname{erfc}\left\{\frac{x-U t(1+\omega)^{1 / 2}}{2 \sqrt{D t}}\right\} \\
\left.+\exp \left\{\frac{U x}{2 D}\left[1+(1+\omega)^{1 / 2}\right]\right\} \operatorname{erfc}\left\{\frac{x+U t(1+\omega)^{1 / 2}}{2 \sqrt{D t}}\right\}\right]
\end{array}\right.
$$


Substitution of J, after simplification of the quantity before the square bracket gives the final solution as:

$$
\frac{C(x, t)}{C_{o}}=\left(\frac{1}{2}\right)\left[\begin{array}{l}
\exp \left\{\frac{U}{2 D} x\left[1-(1+\omega)^{1 / 2}\right]\right\} \operatorname{erfc}\left\{\frac{x-U t(1+\omega)^{1 / 2}}{2 \sqrt{D t}}\right\} \\
+\exp \left\{\frac{U x}{2 D}\left[1+(1+\omega)^{1 / 2}\right]\right\} \operatorname{erfc}\left\{\frac{x-U t(1+\omega)^{1 / 2}}{2 \sqrt{D t}}\right\}
\end{array}\right]
$$

Where:

\subsection{Steady Solution}

$$
\omega=\frac{4 \phi K D}{U^{2}}
$$

For steady state conditions, Eq. (24a) becomes:

$$
\frac{D}{\phi} \frac{\partial^{2} C}{\partial x^{2}}-\frac{U}{\phi} \frac{\partial C}{\partial x}-\frac{K}{\phi} C=0
$$

Thus:

$$
\begin{gathered}
\omega \frac{d^{2} C}{d x^{2}}-\Omega \frac{d C}{d x}-\delta C=0 \\
\omega=\frac{D}{\phi} \\
\Omega=\frac{U}{\phi} \\
\delta=\frac{K}{\phi}
\end{gathered}
$$

Using the characteristic equation involving the variable $\mathrm{m}$, the solution is given as:

$$
C(x)=A \exp \left(\frac{\Omega}{2 \omega} x+\frac{x}{2} \sqrt{\frac{\Omega^{2}}{\omega^{2}}+4 \frac{\delta}{\omega}}\right)+B \exp \left(\frac{\Omega}{2 \omega} x-\frac{x}{2} \sqrt{\frac{\Omega^{2}}{\omega^{2}}+4 \frac{\delta}{\omega}}\right)
$$

Using a similar argument regarding the boundary condition Eq. (23c), the solution is given as:

$$
C(x)=B \exp \left(\frac{\Omega}{2 \omega} x-\frac{x}{2} \sqrt{\frac{\Omega^{2}}{\omega^{2}}+4 \frac{\delta}{\omega}}\right)
$$

Hence:

$$
C(x)=C_{o} \exp \left(\frac{\Omega}{2 \omega} x-\frac{x}{2} \sqrt{\frac{\Omega^{2}}{\omega^{2}}+4 \frac{\delta}{\omega}}\right)
$$




\subsection{Effect of Linear Adsorption Model on Minimum Pressure Drop Required for Foam Generation}

Putting Eq. (2), Eq. (3), Eq. (5) and Eq. (25), it becomes easy to see the explicit dependence of the surface tension of surfactant solution on surfactant concentration and hence the dependence of the minimum pressure drop expressed by Eq. (2), on space and time in the aquifer domain during the surfactant solution injection phase.

\section{Application of Analytical and Numerical solutions to Practical Field Problems}

\subsection{Core Characteristics}

The application of the analytical solution to a practical field problem depends on the availability of data regarding injection rate, surfactant diffusivity in brine, the linear adsorption constant and the petrophysical properties of the porous system, namely porosity and permeability. In this regard, Memon et al., (2017), studied a new natural surfactant found in oil deposits in Iran. The following data can be extracted from their published work:

Table 1. Core and surfactant (Memon, Elraies, \& Al-Mossawy, 2017)

\begin{tabular}{ll}
\hline Parameter & Value \\
\hline Coe length $-\mathrm{cm}$ & 7.66 \\
Core diameter-cm & 3.81 \\
Injection rate of surfactant solution- $\mathrm{cm}^{3} \mathrm{~min}^{-1}$ & 0.24 \\
Number of pore volumes of surfactant solution- $\mathrm{cm}^{3}$ & 0.3 \\
Permeability- $\mathrm{mD}$ & 165 \\
Porosity-fraction & 0.19 \\
Area of core-cm & 11.34 \\
Interfacial tension between injection water and $0.6 \%$ of AOS & 1.24 \\
$+0.6 \%$ LMDO surfactant formulation & \\
\hline
\end{tabular}

\subsection{Parameters of the Linear Model}

Mohsen et al., (2013) also studied Zyziphus Spina Christi obtained from a biological source by hydro distillation of wild leaves. They conducted a static adsorption test based on an outcrop of sandstone samples. Appendix 3 shows their fitted graph to the linear model. Based on this appendix a maximum adsorption of $24 \mathrm{mg} / \mathrm{gm}$ of rock was obtained with a linear adsorption coefficient of $0.199 \mathrm{Lm}^{-2}$ with regression coefficient of 0.978 , which is quite satisfactory. Hence, to link surfactant concentration to interfacial tension and minimum pressure drop required for foam generation, the data of Memon et al., (2017) and the linear adsorption model of Mohsen et al. (2013) with a maximum adsorption of $24 \mathrm{mg} / \mathrm{gm}$ will be integrated for computational purposes. In this regard, data on core dimensions and petrophysical properties will be taken from the former while data on adsorption characteristics will be taken from the latter. The motivation for this decision stems from the fact that petrographic examination of the core samples used by Mohsen et al., (2013) shows abundance of quartz and dolomite minerals, which are similar to those of cores used by Memon et al., (2017). A surfactant concentration of an initial surfactant concentration of $0.5 \mathrm{M}$, will be assumed for computation.

\subsection{Computation of Relevant Parameters}

The final solution to the model equation (48b) contains parameters that deserve to be computed for theoretical testing. They are the following:

$U=$ The superficial velocity of fluid in porous media-ms ${ }^{-1}$

$D=$ Dispersivity $-\mathrm{m}^{2} \mathrm{~s}^{-1}$

\subsection{Choice of Dispersion Coefficient}

Unfortunately, none of the literature sources from which the above data were taken has information about dispersivity. However, in the literature, two theoretical developments have appeared for predicting dispersion coefficient of porous media (Ramirez et al., 1980). By modeling the rock as a bundle of parallel capillary tube, Taylor found the dispersion coefficient to be proportional to pore velocity to the second power (Taylor, 1953). Also, by modeling the rock as a series of connected mixing chambers of uniform size, Aris and Amundson (Aris \& Amundson, 1957) found the dispersing coefficient to be proportional to pore velocity.

Ramirez, et al., (1980), performed a single-phase tracer tests with Berea sandstone core samples with permeability 810 $\mathrm{md}$ and porosity $22 \%$. They concluded from their experimental results that for flow rates in the rage of $10^{-3}-10^{-1} \mathrm{cc}$ per second, mechanical mixing effects are dominant and diffusive, or tortuosity effects are negligible. Appendix 1 shows their experimental results presented as dispersivity versus flow rate. The flow rate given in Table 1 corresponds to $3.33 * 10^{-3}$ cc per second. From Table 2, the porosity of 0.19 is quite closer to $22 \%$. Based on Appendix 1, the dispersion coefficient in light of the given flow rate is $4 * 10^{-8} \mathrm{~m}^{2} \mathrm{~s}^{-1}$. To test the suitability of Appendix 1 for the selection of dispersivity, a dispersion coefficient based on the conclusion of Aris and Amundson (1957 will be computed in the 
following manner:

Step 1: Calculate the mean pore radius using Leveret approach Zhou et al., (2012)

Step 2: Calculate the interstitial velocity as the ratio of superficial velocity to porosity

Step 3: Multiply interstitial velocity by mean pore radius to get mean dispersivity

In this regard, the constant of proportionality is assumed to be equal to the mean pore radius. The computed value of dispersivity is $1.0^{*} 10^{-7} \mathrm{~m}^{2} \mathrm{~s}^{-1}$. This value is quite close to that predicted by Appendix 1

Table 2. Computations based on Literature data (Memon et al., (2017)

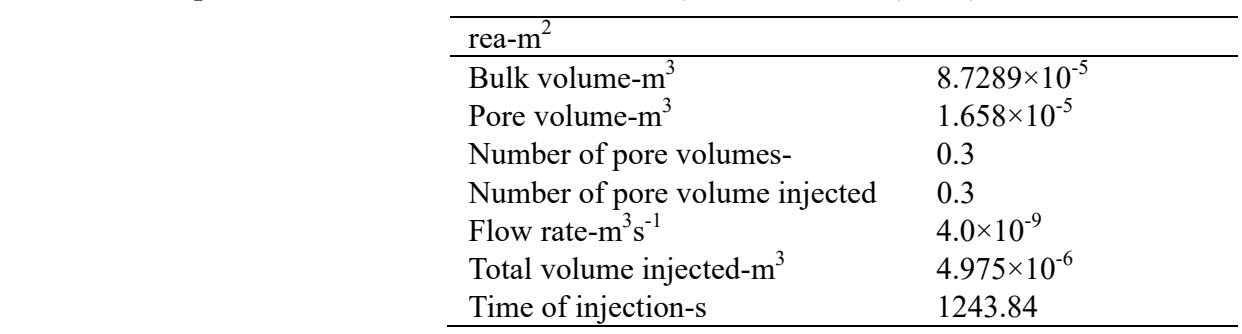

\subsection{Derivation of the Reaction Rate Constant}

From the Linear Model of Memon et al., (2017) the following can be written:

$$
\Gamma=K^{*} C
$$

The linear model used in the derivation of Eq. (21) is:

$$
\frac{d \Gamma}{d t}=K C
$$

Where:

$\Gamma=$ Adsorption-moles per volume of rock

$K=\mathrm{s}^{-1}$

$C=$ Concentration-moles per liter

This means dividing the right-hand side Eq. (33), which is in milligrams per gram of rock by the molecular weight of saponin, we get the number of moles of surfactant adsorbed. If this figure is further multiplied by the density of rock, we get the number of moles of surfactant adsorbed per unit volume of rock. This is the same as Eq. 20 and has concentration unit. If the result is divided by the time of surfactant injection, we get an equation that expresses that expresses rate of change of mass and or concentration. To carry out this calculation, the density of calcite was taken to be $1271 \mathrm{kgm}^{-3}$. The molecular weight of saponin was taken from the work of (Bozicevic et al., 2017). The value is $943.54 \mathrm{~g}$ per mole with the following formula:

$$
\mathrm{C}_{48} \mathrm{H}_{97} \mathrm{O}_{18}
$$

The result gives the Following Equation:

$$
\Gamma=\frac{0.199 * 2710 * 10^{-3}}{1244}=4.595 * 10^{-7} * C
$$

Equation (55) gives the adsorption rate constant equal to $4.595 \times 10^{-7} \mathrm{~s}^{-1}$, considering the linear adsorption model used for the derivation of the advective diffusive equation.

\subsection{Computation of Other Hydraulic Parameters}

Hydraulic parameters required for computations using Eq. (25) are the dispersivity, porosity, permeability and superficial velocity and the respective rations of parameters. Porosity and permeability are found in Tab.1. Dispersivity has already been determined using Appendix 1. Calculated parameters are found in appendix 2 (calculated parameters) 


\subsection{Derivation of Surface Tension versus Distance and Calculation of Minimum Pressure Drop Required for Foam Generation}

To calculate the minimum pressure drop required for foam generation as given by Eq. (2), the effect of surfactant concentration on water or aqueous solution surface tension must be addressed. The solution to the problem requires calculation of the surface tension of surfactant solution as a function of surfactant concentration considering adsorption changes with distance.

Assuming an initial surfactant concentration of $0.5 \mathrm{M}$, the rigorous approach of Yu et al., (2000) was employed to calculate the initial surface tension of surfactant solution. The value is $0.052 \mathrm{mNm}^{-1}$. Equation (55) was then used to calculate adsorption based on the linear model. Calculated adsorption in milligram per grams of rock was then used to calculate surface tension versus corresponding surfactant concentration. The equilibrium or maximum adsorption parameter of Ziziphus Christis found in Eq. (3) was taken from the experimental plots of Mohsen et al., (2013) (See Appendix 3). Once surface tension of aqueous solution as a function of concentration and distance is known, calculation of the minimum pressure drop required for foam generation versus distance and time was achieved using Eq. (2).

\subsection{Results and Discussion}

In the literature, more complex adsorption models, notably the bilinear model have been used to predict adsorption in porous media (Gupta \& Greenkorn, 173)). This paper adopts a rather simplified approach by invoking the linear model, since industrial experiences show that surfactants are generally employed at low concentrations for tertiary oil recovery projects (Rosen et al., 2995). The basis for the application of the linear adsorption model parameters to the solution of the model differential equation under dynamic conditions in this paper is instant adsorption equilibrium. What is more, the dynamic adsorption capacity assumes that only that part of a bed that is called the Equilibrium Zone (EZ) is saturated while the part called the mass transfer zone is under saturated (GROUP, 2010) and this distinction applies mostly to gas adsorption. In the case of surfactant adsorption, the adsorbing phase is non-gaseous. On this note, the parameters of the static model must be similar to that of a dynamic adsorption experiment. In the application of the analytical solution, data from literature sources were chosen objectively and systematically. For instance, justifying the choice of the linear model, it is appropriate to mention the scholarly work of (Ogata, 1961) where the linear model related to adsorption was central to the technical work. What is more, the parameters of the linear model used in this paper were derived using samples of the geologic material. Generally, the degree of adsorption is determined from bench scale experiments consisting typically of mixing contaminant samples with aquifer or rock materials, and measuring adsorption thereof, based on standard procedures (Cheremisinoff, 2017). The literature-based chosen linear model for the present work, therefore, has a scientific validity. I assumed an initial concentration of surfactant solution to be $0.5 \mathrm{M}$.

Fig. 3 shows transient state plots of surfactant concentration versus time for different times of injection of surfactant solution into the core sample. The plots reveal a general decrease of concentration with distance for all times of injection as well as a general increase of concentration with distance as time of injection progresses. During the transient state, the breakthrough time occurs after 4146.44 seconds. 


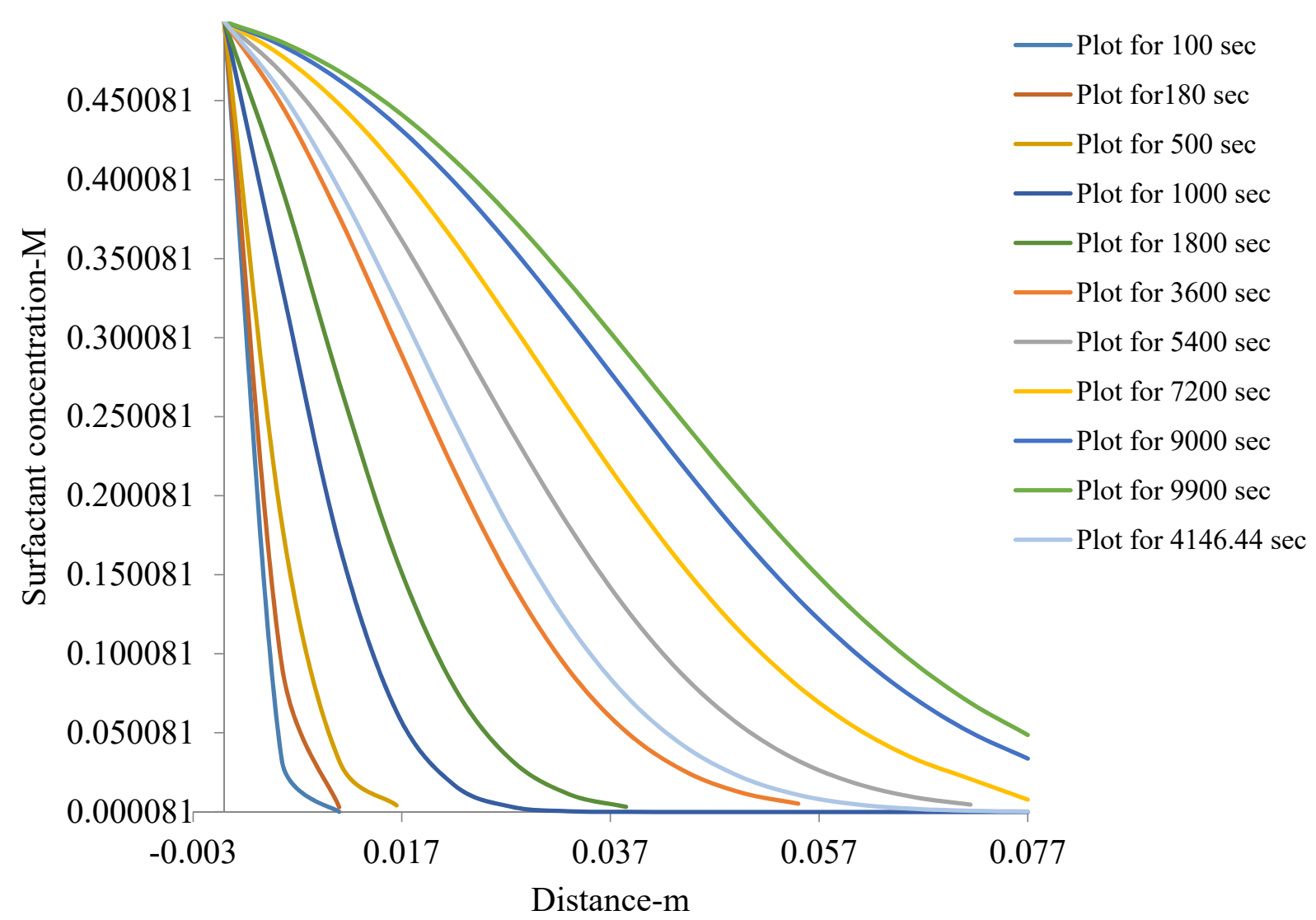

Figure 3. A plot of surfactant concentration versus distance along core

Fig. 4 shows a plot of surfactant concentration versus distance obtained from the solution of the differential equation for steady state conditions (Eq.54). The time steady occurs after 120 days of continuous injection.

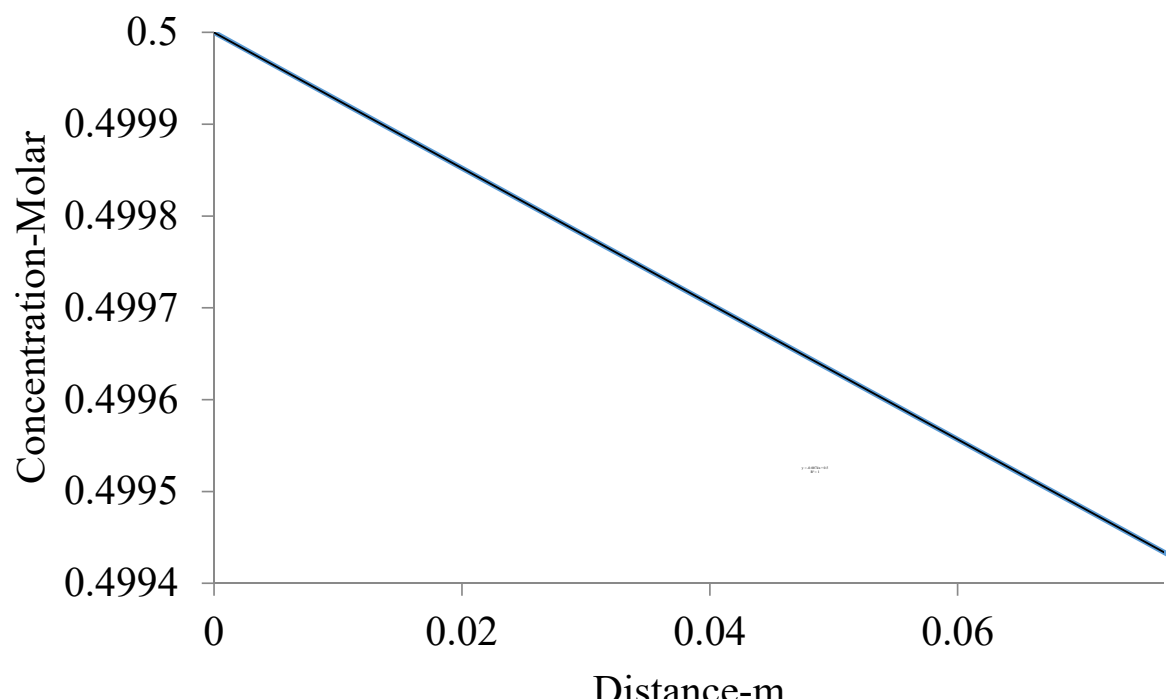

Figure 4. A plot of surfactant concentration versus distanced for steady state

Generally, the surface tension of brine will decrease with increasing surfactant concentration until the critical micelle concentration is reached (Anna et al., 2012). This means as adsorption of surfactant occurs, the surface tension of aqueous solution will increase with distance from the point of injection. Within the frame work of intermolecular forces effect on interfacial tension (Oss et al., 1988), an increase in surfactant surface tension with distance means an overall 
increase in the interfacial tension between injected gas and resident aqueous solution of surfactant brine, following the initial stage of surfactant injection into the aquifer, porous system to displace resident formation brine. With a decrease in mean pore radius due to adsorption coating of pore walls (Renshaw et al., 1997) and increase in interfacial tension with distance from the point of injection, the minimum pressure drop required for foam generation will increase with distance at all times of injection, including the steady state conditions. In all cases, a minimum value of the minimum pressure drop will be encountered at the point of injection while the maximum value will occur at the aquifer boundaries and this trend is clearly shown by Fig. 5, which shows a plot of surface tension versus distance at all times of injection.

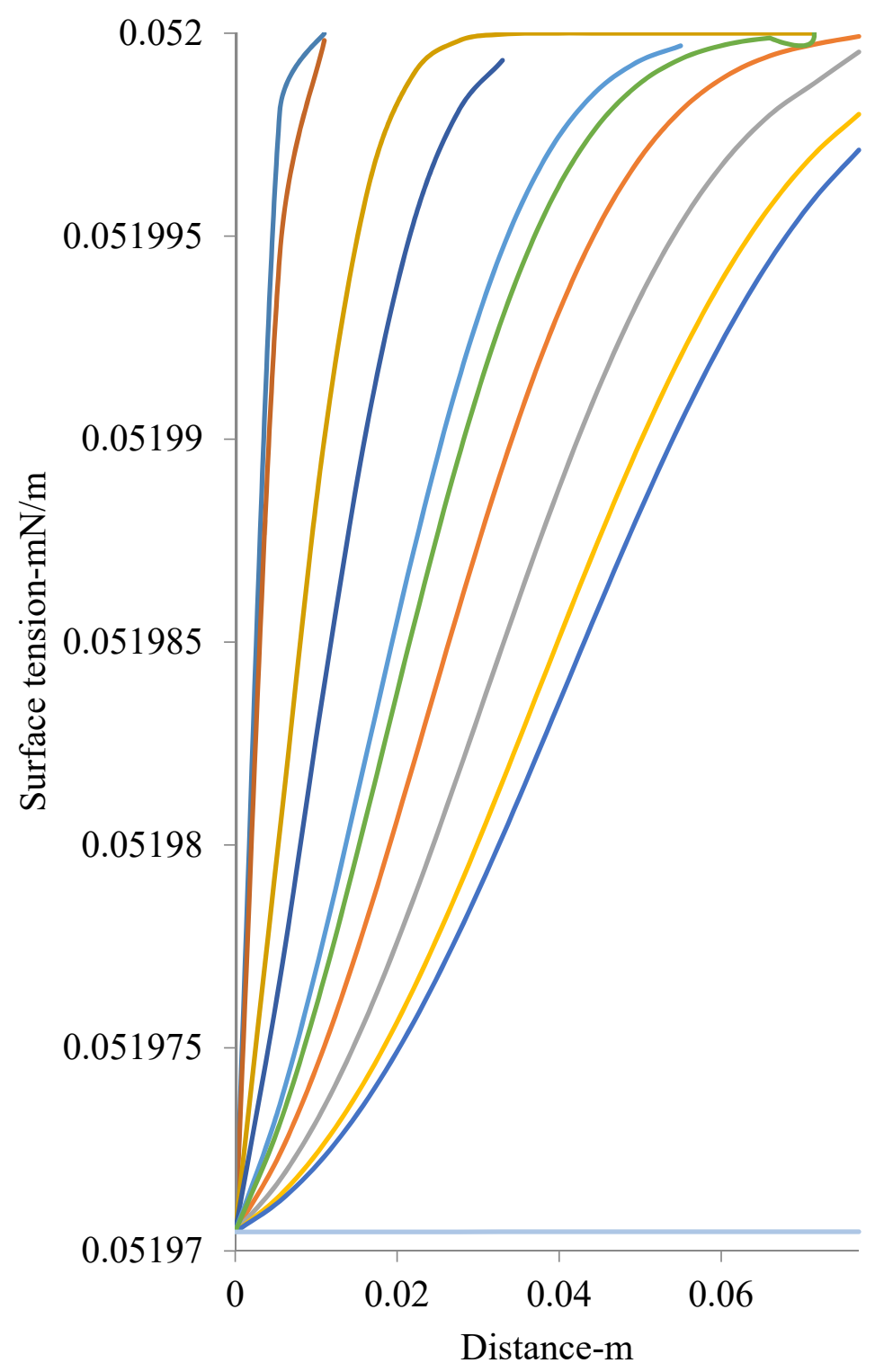

- Surface tension profile for $100 \mathrm{sec}$

- Surface tension profile $180 \mathrm{sec}$

- Surface tension profile for $500 \mathrm{sec}$

- Surface tension profile for $1000 \mathrm{sec}$

- Surface tension profile for $1800 \mathrm{sec}$

- Surface tensionprofile for $3600 \mathrm{sec}$

- Surface tension profile for $3600 \mathrm{sec}$

- Surfacetension profile for $5400 \mathrm{sec}$

- Surface tension profile for $7200 \mathrm{sec}$

- Surface tension profile for $9000 \mathrm{sec}$

- Surface tension profile for $9900 \mathrm{sec}$

Figure 5. A plot of surface tension versus distance for different times of injection

Considering Eq. (2), the minimum pressure drop required for foam generation in a porous system is a function of rock and fluid properties, namely, interfacial tension between injected gas and surfactant solution in the aquifer, the length of the porous system, porosity and permeability. The square root of the ratio of porosity to permeability is the reciprocal of mean sediment pore radius in accordance with Leverets dimensionless capillary pressure function (Zhou et al., 2012). Thus, as adsorption of surfactant occurs, there will be a decrease in mean pore radius with a corresponding increase in pressure drop. Fig.6, which shows a plot of minimum pressure drop required for foam generation for different times of injection reveals the pressure increase trend. 


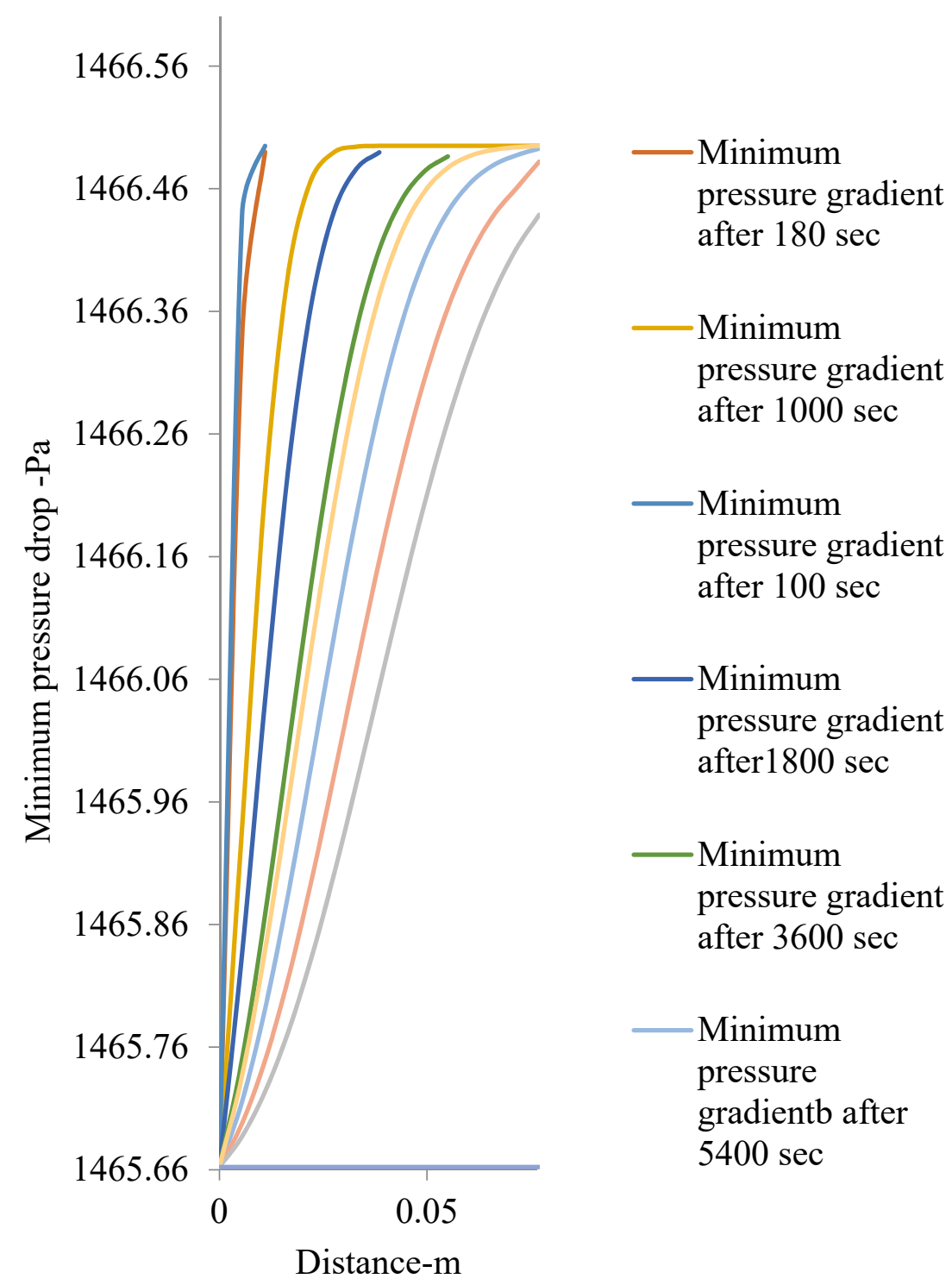

Figure 6. A plot of minimum pressure drop versus distance for different times of injection

Figure 6shows a plot of surface tension of injected surfactant solution versus concentration at steady state conditions of surfactant injection. The plot shows a linear relationship. In the literature, a plot of surface tension versus the logarithm of surfactant concentration is known to give a linear relationship (Lin et al. 1999). Therefore, the trend shown by Fig.7, where the horizontal axis is linear contradicts theory. 


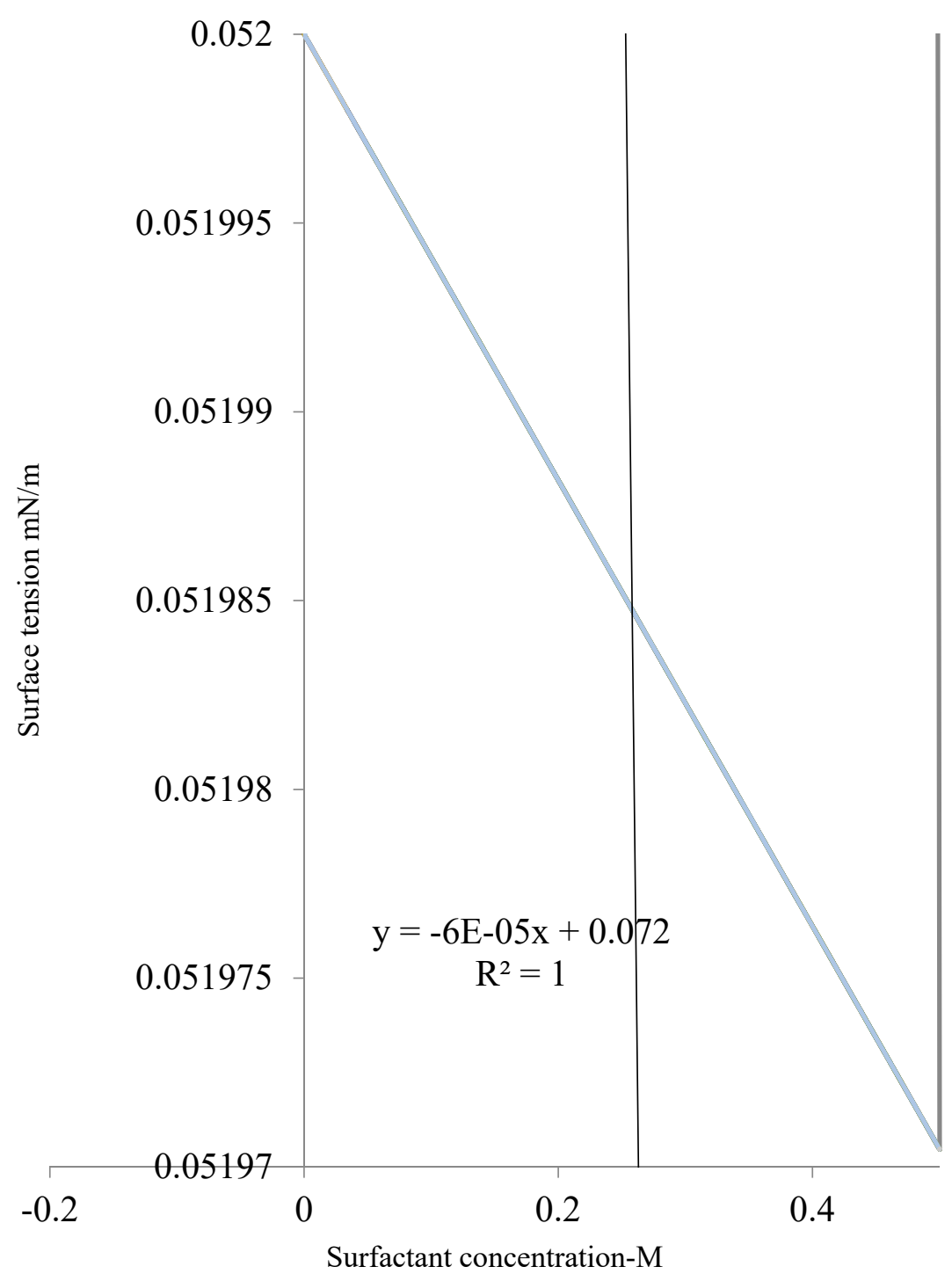

Figure 7. A plot of surface tension versus surfactant concentration at steady state conditions

However, considering Eq. (3), where surface tension is linked to adsorption, the trend given by Fig.7 can be approximated in accordance with the following mathematical argument:

Equation 3 gives the following:

$$
\sigma-\sigma_{0}=R T \Gamma^{\infty} \ln \left(1-\frac{\Gamma}{\Gamma^{\infty}}\right)
$$

The linear adsorption model gives the following equation:

$\Gamma=K C$

Where all parameters have their usual meanings:

Substitution of the second equation into the first equation for adsorption gives the following equation:

$$
\sigma-\sigma_{0}=R T \Gamma^{\infty} \ln \left(1-\frac{K C}{\Gamma^{\infty}}\right)=R T \Gamma^{\infty} \ln (1+\delta C)
$$

Where: 


$$
\delta=\frac{K}{\Gamma^{\infty}}
$$

In the first place, Eq. (36) shows that at maximum adsorption, the following can be written:

$$
\begin{aligned}
& \sigma-\sigma_{0}=R T \Gamma^{\infty} \ln \left(1-\frac{K C}{\Gamma^{\infty}}\right)=R T \Gamma^{\infty} \ln (1-1)=\ln (0)=0 \\
& \Rightarrow \sigma=\sigma_{o}
\end{aligned}
$$

This is to be expected because at maximum adsorption, there is no more adsorption as injection continues so the concentration of surfactant solution at every point in the porous medium must be equal to the initial concentration.

Using series expansion, the right-hand side of Eq. (36) can be expanded as:

$$
\begin{aligned}
& \sigma-\sigma_{0}=R T \Gamma^{\infty} \ln \left(1-\frac{K C}{\Gamma^{\infty}}\right)=R T \Gamma^{\infty} \ln (1+\delta C)=-\delta C-0.5 \delta^{2} C^{2}-0.333 \delta^{3} C^{3} \\
& -0.25 C^{4}-0.2 \delta^{5} \ldots .
\end{aligned}
$$

Considering the linear model, which assumes lower concentrations, powers of concentration above 2 may be neglected to give the following equation after solving for surface tension:

$$
\sigma=-\delta C+\sigma_{0}
$$

Based on Eq. (40), the linear plot of surface tension versus surfactant concentration at steady state conditions as shown by Fig. 7 is to be expected with a negative gradient and initial surface tension as predicted in this equation.

Generally, Fig. 3, Fig. 5 and Fig. 6 show gradual changes in gradients with time. Ziziphus Christis is a new biosurfactant, also found in association with petroleum deposits. Being a surfactant of biological origin in addition to its presence in petroleum deposits, the potential for its use in tertiary oil recovery projects like conventional ones has been explored in the literature. Consequently, adsorption experiments, where crushed rock samples have been contacted with solutions of Ziziphus Christis have been performed in the literature. Accordingly, adsorption of this surfactant has been found to obey a linear model. In this paper, I have used the linear model of adsorption to derive a mass balance in the form of linear partial differential equation of second order. I have solved the equation based on physically realistic boundary conditions. Based on the solution, I have used a linear adsorption data from literature to determine numerical constants of the solution to the differential equation. Assuming an initial surfactant concentration 0.5 Molar, I have calculated concentration versus distance for adsorption onto a calcite core sample of specified porosity, permeability and length. Superficial velocity was determined based on injection rate found in the literature. Dispersivity was deduced from Appendix 1.

\subsection{Conclusion}

While several studies have appeared regarding the adsorption of Ziziphus Christis on reservoir rock samples, the present study is the first to present analytical calculation solution to advective-dispersive transport of Ziziphus Christis in a porous medium with a linear adsorption model. The following sums up the conclusion of this study:

1. The dynamic adsorption behavior of Ziziphus Christis shows a gradual decrease in gradient with time of injection, which is indicative of a gradual increase of surfactant concentration in brine with time in the porous medium.

2. This trend in adsorption, considering advective-dispersive transport in porous media with a linear adsorption model has been reported elsewhere (Ogata, 1961)

3. In terms of the minimum pressure required for foam generation in porous medium, the minimum pressure drop will increase with distance at all times of injection and become time independent during the steady state period. 
4. Considering the appreciably low surfactant concentration used in this study, the biosurfactant can be said to have a foamability potential similar to traditional surfactants found in the petroleum industry

5. Given that the analytical solution to the model differential equation uses parameters of the linear adsorption model reported from literature in connection with adsorption experiments using Ziziphua Christis, the present work based on dynamic adsorption is an extension of the investigation of this surfactant not only for enhanced oil recovery but also for surfactant enhanced geological storage projects.

\section{Appendices}

Appendix 1: Dispersion coefficient versus phase velocity (Ramirez, Shuler, \& Friedman, 1980),

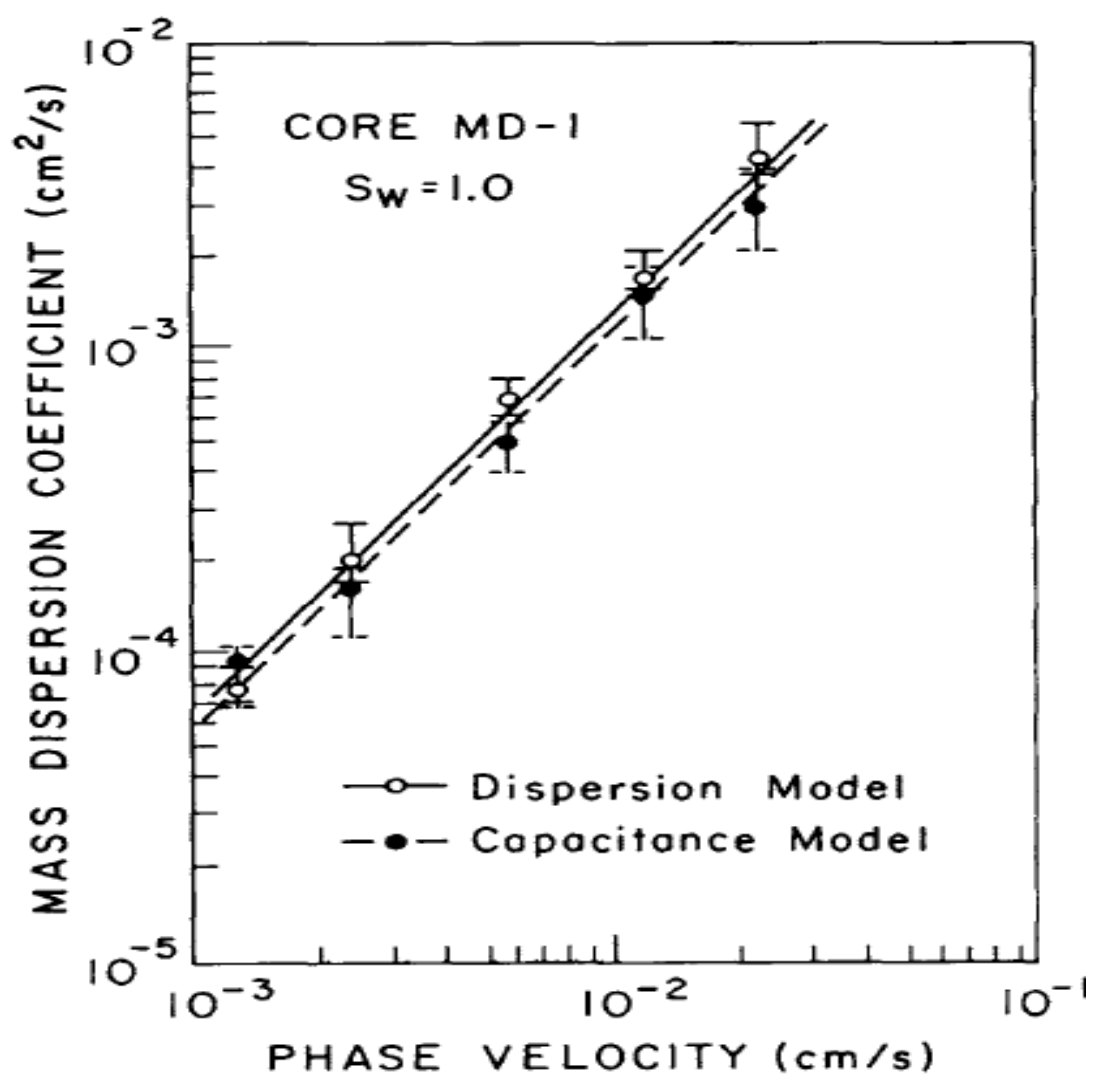


Appendix 2: Calculated Parameters Based on Literature Data

$$
\begin{aligned}
& U=3.51 * 10^{-6} \mathrm{~ms}^{-1} \\
& D=4 * 10^{-8} \mathrm{~m}^{2} \mathrm{~s}^{-1} \\
& \frac{U}{D}=87.75 \mathrm{~m}^{-2} ? \\
& \frac{U^{2}}{D^{2}}=7700 \mathrm{~m}^{-2} \\
& K=5.251 * 10^{-8} \mathrm{~s}^{-1} \\
& \frac{\delta}{\omega}=\frac{K}{D}=1.131 \mathrm{~m}^{2} \\
& \frac{\Omega}{\omega}=\frac{U}{D}=87.75 \mathrm{~m}^{-1} \\
& \mu, \omega ?=6.82 * 10^{-5}
\end{aligned}
$$

Appendix 3: Adsorption with maximum value (Mohsen et al., 2013)

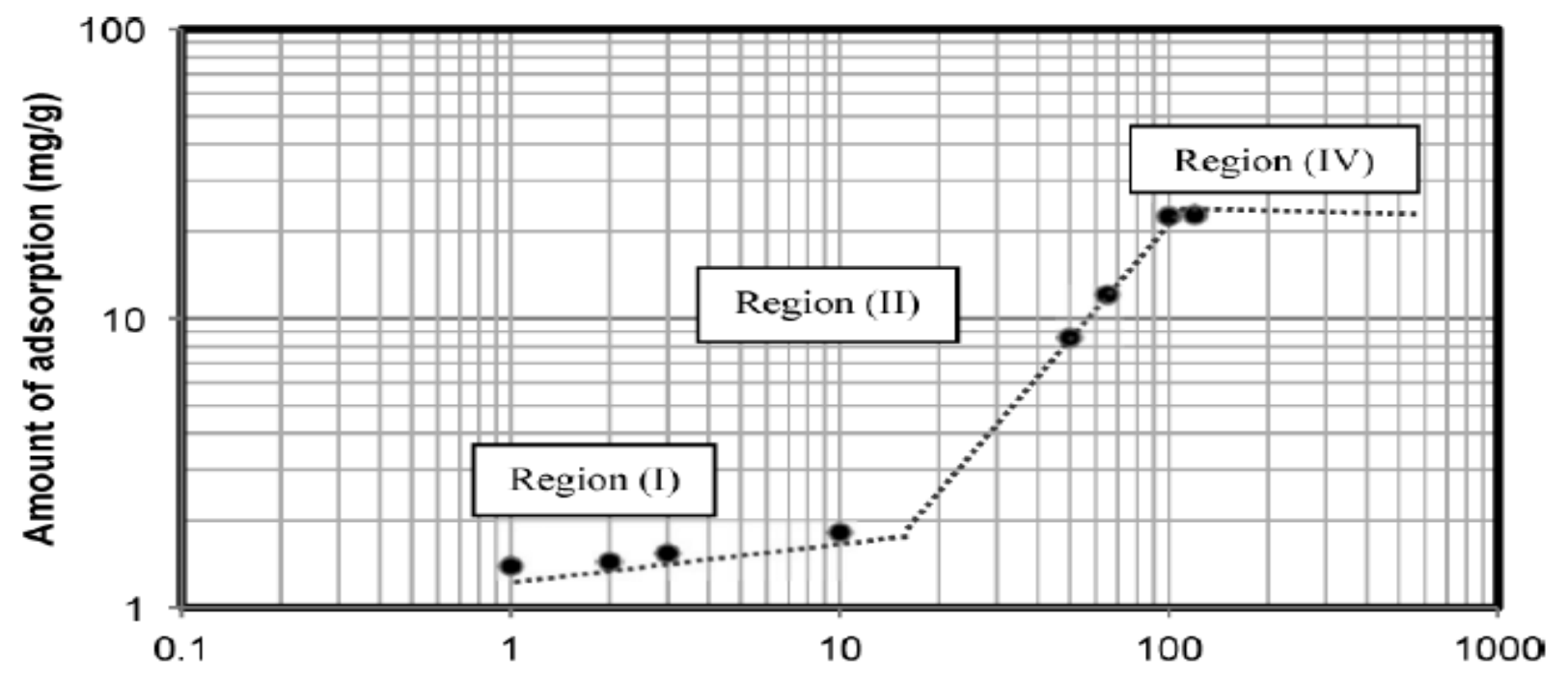

\section{References}

Abdel-Zaher, A., Salim, S., Assaf, M., \& Abdel-Hady, R. H. (2005). Antidiabetic activityand toxicity of Zizyphus spina-christi leaves. J. Ethnopharmacol, 101, 129-138. https://doi.org/10.1016/j.jep.2005.04.007

Aghdam, K. A., Moghaddas, J. S., Moradi, B., Dabiri, M., \& Hassanzadeh, M. (2013). Maximizing the Oil Recovery ThroughMiscible Water Alternating Gas (WAG)Injection in an Iranian Oil Reservoir. Petroleum Science and Technology, 31, 2431-2440. https://doi.org/10.1080/10916466.2011.569822

Al-Rbeawi, S. A., \& Tiab, D. (2014). Impacts of reservoir boundaries and fracture dimensions on pressure behaviors 
and flow regimes of hydraulically fractured formations. Journal of Petroleum Exploration and Production Technology, 4(1), 37-57. https://doi.org/10.1007/s13202-013-0070-1

Anna, Z., Szymczyk, K., \& Jańczuk, J. K. (2012). Critical micelle concentration of some surfactants and thermodynamic parameters of their micellization. Fluid Phase Equilibria, 322-323(25), 126-134.

Apaydin, O., \& Kovscek, A. (2001). Surfactant Concentration and End Effects on Foam Flow in Porous Media. Transport in Porous Media, 43, 511-536. https://doi.org/10.1023/A:1010740811277

Aris, R., \& Amundson, N. (1957). Some Remarks on Longitudinal Mixing or Diffusion in Six Beds. AIChE J., 3, 280-282. https://doi.org/10.1002/aic.690030226

Bachu, S. (2000). Sequestration of CO2 in geological media: criteria and approach for site selection in response to climate change. Energy Conversion and Management, 41(9), 953-970. https://doi.org/10.1016/S0196-8904(99)00149-1

Bachu, S., \& Bennion, D. B. (2009). nterfacial Tension between CO2, Freshwater, and Brine in the Range of Pressure from (2 to 27) $\mathrm{MPa}$, Temperature from $(20$ to 125$){ }^{\circ} \mathrm{C}$, and Water Salinity from (0 to 334000$) \mathrm{mg} \cdot \mathrm{L}-1$. J. Chem. Eng. Data, 54(3), 765-775. https://doi.org/10.1021/je800529x

Barati, A., Njafe, A., Daryasafi, A., Nadali, P., \& Moslehi, H. (2016). Adsorption of a new nonionic surfactant oncarbonate minerals in enhanced oil recovery:Expoerimental and Modeling Studies. Chemical Engineering Research and Design, 105, 55-63. https://doi.org/10.1016/j.cherd.2015.10.047

Bozicevic, A., De Mieria, M., Di Benedetto, A., \& Gafner, F. (2017). Dammarane-type saponins from leaves of Ziziphus spina-christi. Phytochemistry, 138, 134-144. https://doi.org/10.1016/j.phytochem.2017.02.028

Bozicevic, A., Mieri, M. D., Benedetto, A. D., \& Gafner, F. (2017 ). spina-christi, Dammarane-type saponins from leaves of Ziziphus. Phytochemistry, xxx, 1-11. https://doi.org/10.1016/j.phytochem.2017.02.028

Bozicevic, A., Mieri, M. D., Benedetto, A. D., \& Gafner, F. (2017). Dammarane-type saponins from leaves of Ziziphus spina-christi. Phytochemistry, 1-10. https://doi.org/10.1016/j.phytochem.2017.02.028

Cameron, D. R. (1977). Convective-dispersive solute transport with a combined equilibrium and kinetic adsorption model. Water Resources Research, 13(1), 183-188. https://doi.org/10.1029/WR013i001p00183

Chapter5. (n.d.). Retrieved from Fundamentals of Fluid Flow in Porous Media: http://perminc.com/resources/fundamentals-of-fluid-flow-in-porous-media/chapter-5-miscible-displacement/equati on-continuity-porous-media/convection-dispersion-model-adsorption/

Chen, D. L., \& Al-Saidi, W. A. (2012). The role of van der Waals interactions in the adsorption of noble gases on metal surfaces. Journal of Physics: Condensed Matte, 24(42), 1-10. https://doi.org/10.1088/0953-8984/24/42/424211

Chen,'X. Y., Kianinejad, A., \& DiCarlo, D. A. (2014). An Experimental Study of CO2-Brine Relative Permeability in Sandstone. SPE Improved Oil Recovery Symposium, 12-16 April, Tulsa, (pp. 1-14). Society of Petroleum Engineers. https://doi.org/10.2118/169137-MS

Chena, M., Yortsos, C., \& Rossen, W. (2005). Insights on foam generation in porous media from pore-network studies. Colloids and Surfaces A: Physicochem. Eng. Aspects, 256, 181-189. https://doi.org/10.1016/j.colsurfa.2005.01.020

Cheremisinoff, N. P. (2017). Groundwater Remediation A Practical Guide for Enviromental Engineers and Scientists. Wiley. https://doi.org/10.1002/9781119407621

Chrysikopoulous, C. V., Kitanidis, P. K., \& Roberts, P. V. (1990). Analysis of One Dimensional Solute Transport Through Porous Media with Sapatially Variable Retardation. Water Resources Research, 26(3). https://doi.org/10.1029/WR026i003p00437

Didier, P. (1992). Observations and Correlations for Immiscible Viscous-Fingering Experiments. Reservoir Engineering, 7(2), 187-194. https://doi.org/10.2118/19670-PA

Do, D. D., \& Rice, R. G. (2012 ). Applied Mathematics and Modeling for Chemical Engineers. John Wiley \& Sons.

Eastoe, J., \& Dalton, J. (2000). Dynamic surface tension and adsorption mechanisms of surfactants at the air-water interface. Advances in Colloid and Interface Science, 85(2-3), 103-144. https://doi.org/10.1016/S0001-8686(99)00017-2

Fabíola, D. C., C., V., L., S., Neto, B., Jr., T. V., CastroDantasb, T. N., ... Garnicac, A. (2007). Adsorption of nonionic surfactants in sandstones. Colloids and Surfaces A: Physicochemical and Engineering Aspects, 293(1-3), 1-4. https://doi.org/10.1016/j.colsurfa.2006.06.038 
Farokhpoor, R., Bjørkvik, B. J., Lindeberg, E., \& Torsætera, O. (2013). CO2 Wettability Behavior During CO2 Sequestration in Saline Aquifer -An Experimental Study on Minerals Representing Sandstone and Carbonate. Energy Procedia, 37, 5339-5351. https://doi.org/10.1016/j.egypro.2013.06.452

Frank, D. G., Katekaru, J. Y., Rosasco, S. D., Salaita, G. N., Schardt, B. C., Soriaga, M. P., ... Hubbard, A. T. (1985). pH and potential dependence of the electrical double layer at well-defined electrode surfaces: $\mathrm{Cs}+$ and $\mathrm{Ca} 2+$ ions at $\operatorname{Pt}(111)(2 \sqrt{ } 3$ X. 2 $\sqrt{ } .3) R 30^{\circ}-\mathrm{CN}, \operatorname{Pt}(111)(\sqrt{13} \mathrm{X} \sqrt{ } .13) \mathrm{R} 14^{\circ}-\mathrm{CN}$, and $\operatorname{Pt}(111)(2 \mathrm{X} 2)-\mathrm{SCN}$. Langmuir, 1(5), 587-592. https://doi.org/10.1021/la00065a013

Freundlich, H. (1906). Uber die adsorptionin lasungen. Z. Phys. Chem. (Leipzig), 57 A, 385-470.

Gandomkar, A., \& Kharrat, R. (2013). Anionic Surfactant Adsorption through Porous Media in Carbonate Cores: An Experimental Study. Energy Sources, Part A, 35, 58-65. https://doi.org/10.1080/15567036.2010.501368

Gauglitz, P., Friedmann, F., Kam, S., \& Rossenc, W. (2002). Foam generation in homogeneous porous media. Chemical Engineering Science, 57, 4037-4052. https://doi.org/10.1016/S0009-2509(02)00340-8

Gildnyi, T., Stergiopoulos, C., \& Wolfram, E. (1976). Equilibrium surface tension of aqueous surfactant solutions. Colloid \& Polymer Sci., 254, 1018-1023. https://doi.org/10.1007/BF01516920

Global Warming, I. (2009). CO2-the major cause of global warming. Retrieved from http://timeforchange.org/CO2-cause-of-global-warming

Gogoi, S. B. (2011). Adsorption-Desorption of Surfactant for Enhanced Oil Recovery. Transport in Porous Media, 90, 589-604. https://doi.org/10.1007/s11242-011-9805-y

Group, C. A. (2010). Static and Dynamic Asorption Capacity . Retrieved from www.Siliporite.com

Gu, T., Rupprecht, H., \& Galera, P. A. (1993). Colloid Polym. Sci., 271, 271, 799-801. https://doi.org/10.1007/BF00660098

Gupta, S. P., \& Greenkorn, R. A. (173). Dispersion during flow in porous media with bilinear adsorption. Water Resources Journal, 9(5), 1357-1368 https://doi.org/10.1029/WR009i005p01357

Hirasaki, G., Szafranski, R., Miller, C. A., \& Wade, W. (1977). Field Demonstration of the Surfactant/Foam Process forRemediation of a Heterogeneous Aquifer Contaminated with DNAPL. 1997 SPE Annual Technical Conference and Exhibition held in San Antonio, Texas, 5-8 October 1997, (pp. 1-11).

Huang, D. D., Nikolov, A., \& Wasan, D. T. (1986). Foams: Basic Properties with Application to Porous Media. Langmuir, 2, 673-677. https://doi.org/10.1021/la00071a027

Iglauer, S. (2011). Curtin University, Department of Petroleum Engineering. Retrieved from Dissolution Trapping of: www.intechopen.com

Kantzas, A., Bryan, J., \& Taheri, S. (2016). Chapter 5. In Fundamentals of Fluid Flow in Porous Media. PERM Inc.

Kim, Y., Wan, J., Kneafsey, T. J., \& Tokunaga, T. K. (2012). Dewetting of Silica Surfaces upon Reactions with Supercritical $\mathrm{CO} 2$ and Brine: Pore-Scale Studies in Micromodels. Environmental Science \& Technology, 46, 4228-4235. https://doi.org/10.1021/es204096w

Kwok, W., Hayes, R., \& Nasr-El-Din, H. (1995). Modelling dynamic adsorption of an anionic surfactant on Berea sandstone with radial flow. Chemical Engineering Science, 50, 769-783. https://doi.org/10.1016/0009-2509(94)00450-6

Lapidus, L., \& Amundson, N. (1952). Mathematics of adsorption in Chromatographic Column. Journal of Physisical Chemistry, 56, 984-988. https://doi.org/10.1021/j150500a014

Li, K., Jing, X., He, S., \& Wei, B. (2016). Static Adsorption and Retention of Viscoelastic Surfactant in Porous. Energy Fuels, 9089-9096. https://doi.org/10.1021/acs.energyfuels.6b01732

Lin, S. Y., Lin, Y. Y., Chen, E. M., Hsu, C. T., \& Kwan, C. C. (1999). A Study of the Equilibrium Surface Tension and the Critical Micelle Concentration of Mixed Surfactant Solutions. Langmuir, 15, 4370-4376. https://doi.org/10.1021/la981149f

Lindeberg, E., \& Wessel-Berg, D. (1997). Vertical convection in an aquifer column under a gas cap of CO2. Energy Conversion and Management, 38, 229-234. https://doi.org/10.1016/S0196-8904(96)00274-9

Memon, M. K., Elraies, K. A., \& Al-Mossawy, M. I. (2017). Impact of new foam surfactant blend with water alternating gas injection on residual oil recovery. $J$ Petrol Explor Prod Technol, 7, 7:843-851. https://doi.org/10.1007/s13202-016-0303-1 
Mohsen, S. B., Shari, M. P., Zargartalebi, M., \& Arabloo, M. (2013). New Surfactant Extracted from Zizipus Spina Christi foe Enhanced Oil Recovery: Experimental Determination of Static Adsorption. Journal of Japan Petroleum Institute, 56(3), 142-149. https://doi.org/10.1627/jpi.56.142

Monroe, R. (2013, April). Retrieved from We Just Breached the 410 PPM Threshold for CO2: https://scripps.ucsd.edu/programs/keelingcurve/2015/05/12/what-does-this-number-mean/

Moortgat, J. (2016). Viscous and Gravitational Fingering in Multiphase Compositional and Compressible Flow. Advances in Water Resources , 1-32. https://doi.org/10.1016/j.advwatres.2016.01.002

Müller, N. (2011). Supercritical CO2-Brine Relative Permeability Experiments in Reservoir Rocks-Literature Review and Recommendations. Transport in Porous Media, 87(2), 367-383. https://doi.org/10.1007/s11242-010-9689-2

Nghiem, L., Yang, C., Shrivastava, V., Kohse, B., Hassam, M., \& Card, C. (2009). Risk mitigation through the optimization of residual gas and solubility trapping for $\mathrm{CO} 2$ storage in saline aquifers. Energy Procedia, 1(1), 3015-3022. https://doi.org/10.1016/j.egypro.2009.02.079

Ogata, A. (1964). Mathematics of Dispersion With Linear Adsorption Isotherm :A theoretical consideration of the effect of a reacting media on the dispersion of fluids in porous media. Geological Surevey Professional Paper 411-H.

Ogatas, A., \& Banks, R. B. (1961). A Solution of tghe Differential Equation of Longitudinaql Dispoersion in Porous Media. U.S Geological Survey Professional Paper 411-A, A1-A9.

Oss, C. J., Good, R. J., \& Chaudhury, M. K. (1988). Additive and nonadditive surface tension components and the interpretation of contact angles. Langmuir, 4(4), 884-891. https://doi.org/10.1021/la00082a018

Patil, S., \& Chore, H. (2014). Contaminant transport through porous media: An overview of experimental and numerical studies. Advances in Environmental Research, 1(3), 45-69. https://doi.org/10.12989/aer.2014.3.1.045

Peeters, F., \& Hofmann, H. (2015). Length-scale dependence of horizontal dispersion in the surface water of lakes. Limnol. Oceanogr, 60, 1917-1934. https://doi.org/10.1002/lno.10141

Petrphysiswiki. (n.d.). Retrieved from Petrowiki.org/Filevol//Page_583 Image0001.png

Pordel, S. P., Shadizadeh, S., \& Jamialahmadi, M. (2012). Applicability test of newsurfactant produced from Zizyphus Spina Christi leaves for enhanced oilrecovery in carbonate reservoirs. J Jpn Pet Inst, 55(1), $27-32$. https://doi.org/10.1627/jpi.55.27

Pruess, K., \& García, J. (2002). Multiphase flow dynamics during CO2 disposal into saline aquifers. 282-295, 42(2-3), 282-295. https://doi.org/10.1007/s00254-001-0498-3

Rahmati, M. M. M. (2015). Effect of Natural Leaf-derived Surfactants on Wettability Alteration and Interfacial Tension Reduction in Water-oil System: EOR Application. Japan Petroleum Institute, 4, 245-251. https://doi.org/10.1627/jpi.58.245

Ramirez, W. F., Shuler, P. J., \& Friedman, F. (1980). Convection, Dispersion, and Adsorption of Surfactants in Porous Media. Society of Petroleum Engineers of AIME, 430-438. https://doi.org/10.2118/7951-PA

Renshaw, C. E., Zynda, G. D., \& Fountain, J. C. (1997). Permeability reductions induced by sorption of surfactant. Water Resources Resaerch, 33(3), 371-378. https://doi.org/10.1029/96WR03299

Rosen, M., Wang, H., Shen, P., \& Zhu, Y. (2005). Ultralow interfacial tension for enhanced oil recovery at very low surfactant concentrations. Langmuir, 21(9), Langmuir. 2005 Apr 26; 21(9), 3749-56. https://doi.org/10.1021/la0400959

Rupprecht, H., \& Gu, T. (1991). Colloid Polym. Sci., 269, 506-522. https://doi.org/10.1007/BF00655889

Ruthven, D. (1982). Principles of Adsorption and Adsorption Processes. Wiley Inter-Science, New York, NY.

Safian-Boldaji, M., Mojtaba, Pordel, S., Mohammad, Z., \& Milad, A. (2013). New Surfactant Extracted from Zizyphus Spina-Christi for Enhanced Oil Recovery: Experimental Determination of Static Adsorption Isotherm. Journal of the Japan Petroleum Institute, 56(3), 142-149. https://doi.org/10.1627/jpi.56.142

Schramm, L. (1994). Foams: Fundamentals and applications in the Petroloeum Industry. ACS Advance in Chemical Series(242). https://doi.org/10.1021/ba-1994-0242

Schramm, L. L. (2009). Surfactants: Fundamentals and Applications in the Petroleum Industry. Cambridge: Cambridge University Press.

Shahri, M. P., Shadizadeh, S. R., \& Jamialahmadi, M. (2007). A New Type of Surfactant for Enhanced Oil Recovery. Petroleum Science and Technology, 30(6), 585-593. https://doi.org/10.1080/10916466.2010.489093 
Shariatipour, S. M., Pickup, G., Mackay, E., \& Heinemann, N. (2012). Flow Simulation of CO2 Storage in Saline Aquifers Using Black Oil Simulator. Carbon Management Technology Conference, 7-9 February (pp. 1-13). Orlando, Florida, USA : Carbon Management Technology Conference. https://oi.org/10.7122/151042-MS

Solomon, S. P., Knutti, R., \& Friedlingstein, P. (2009). Irreversible climate change due to carbon dioxide emissions. Proc Natl Acad Sci U S A, 106(6), 1704-1709. https://doi.org/10.1073/pnas.0812721106

Spildo, K., Johannessen, A., \& Skauge, A. (2012). Low salinity waterflood at reducedcapillarity. Low salinity waterflood at reducedcapillarity. In: Eighteenth SPE improved oil recovery symposium. held in Tulsa, Oklahoma: SPE.

Sun, J.-J., Zhu, Q.-Y., \& Yub, H.-Z. (2015). The influence of temperature gradient on the convective mixing phenomena of geologic sequestration of CO2. 7th International Conference on Fluid Mechanics, ICFM7. 126, pp. 421-425. Procedia Engineering. https://doi.org/10.1016/j.proeng.2015.11.259

Taylor, G. J. (1953). Dispersion of Soluble Matter in Solvent Flowing Slowly Through a Tube. Proc., Royal Soc., 219, 186-203. https://doi.org/10.1098/rspa.1953.0139

Temkin, M., \& Pyzhev, V. (1940). Recent Modifications to Langmuir Isotherms. Acta Physiocochim, USSR, 217-222.

Terdre, N. (2003). Foam Assisted Injection Trials Could Spread to other North Sea Fields. Ofshore, 63.

Verma, M. K. (2015). Fundamentals of Carbon Dioxide-Enhanced Oil Recovery (CO2-EOR)—A Supporting Document of the Assessment Methodology for Hydrocarbon Recovery Using CO2-EOR Associated with Carbon Sequestration . Virginia: Department of the Interior ,U.S. Geological Survey Open-File Report 2015-1071 .

Versteeg, H., \& Malalasekera, W. (1995). An Introduction to Computational Fluid Dynamics: The Finite Volume Element. New York: Prentice Hall.

Vitoonkijvanich, S., AlSofi, A. M., \& BluntaaImperial, M. J. (2015). Design of foam-assisted carbon dioxide storage in a North Sea aquiferusing streamline-based simulation. International Journal of Greenhouse Gas Control, 33, 113121. https://doi.org/10.1016/j.ijggc.2014.11.022

Vizquez, G., Cancela, M., Varela, R., Alvarez, E., \& Navaza, J. (1997). Influence of surfactants on absorption of CO2 in a stirred tank with and without bubbling. Chemical Engineering Journal, 67, 131-137. https://doi.org/10.1016/S1385-8947(97)00047-8

Wexler, E. J. (1992). Technique of Water Resources Investigation of The United Staes Geological Survey. Denver: U S Geological Survey.

Yu, Y. X., Ga, G. H., \& Li, Y. G. (2000). Surface tension for aqueous electrolyte solutions by the modified mean spherical approximation. Fluid Phase Equilibria, 173, 23-38. https://doi.org/10.1016/S0378-3812(00)00396-4

Zekri, A. Y., Nasr, M. S., \& Alshobakyh. (2011). Evaluation of Oil Recovery by Water Alternating Gas (WAG) Injection - Oil-Wet \& Water-Wet Systems. SEP Enhanced Oil Recovery in Malaysia, 2011 (pp. 1-8). Kuala Lampur: SPE.

Zendehboudi, S., Ahmadi, M., Rajabzadeh, A., Mahinpey, N., \& Chatzis, I. (2013). .Experimental study on adsorption of a new surfactant onto carbonatereservoir samples-application to EOR. Can J Chem Eng, 1-11. Retrieved from https:// dx.doi.org/10.1002/cjce.2180

Zhou, Y., Helland, J., \& Hatzignatiou, D. G. (2012). A Dimensionless Capillary Pressure Function for Imbibition Derived From Pore-Scale Modeling in Mixed-Wet-Rock Images. Society of Petroleum Engineers, 18(2), 296-303.

Ziska, L. H., \& Caulfield, F. A. (2000). Rising CO2 and pollen production of common ragweed (Ambrosia artemisiifolia L.), a known allergy-inducing species: implications for public health. Australian Journal of Plant Physiology, 27(10), 893 - 898.

Zlotnik, V. A., \& Logan, J. D. (1996). Boundary Conditions for Convergent Radial Tracer Tests and Effect of Well Bore Mixing Volum. Water Resource Research, 32(7), 2323-2328. https://doi.org/10.1029/96WR01103

\section{Copyrights}

Copyright for this article is retained by the author(s), with first publication rights granted to the journal.

This is an open-access article distributed under the terms and conditions of the Creative Commons Attribution license (http://creativecommons.org/licenses/by/4.0/). 